\title{
Higgs and non-universal gaugino masses: no SUSY signal expected yet?
}

\author{
Sascha Caron,,$^{a, b}$ Jari Laamanen, ${ }^{a, b}$ Irene Niessen ${ }^{a, b}$ and Antonia Strübig ${ }^{c}$ \\ ${ }^{a}$ Experimental and Theoretical High Energy Physics, \\ IMAPP, Faculty of Science, Radboud University Nijmegen, \\ Mailbox 79, P.O. Box 9010, NL-6500 GL Nijmegen, The Netherlands \\ ${ }^{b}$ Nikhef, \\ Science Park 105, 1098 XG Amsterdam, The Netherlands \\ ${ }^{c}$ Physikalisches Institut, University of Freiburg, \\ Hermann-Herder Str.3, 79104 Freiburg, Germany \\ E-mail: Sascha.Caron@cern.ch, j.laamanen@science.ru.nl, \\ i.niessen@science.ru.nl, antonia.struebig@physik.uni-freiburg.de
}

ABSTRACT: So far, no supersymmetric particles have been detected at the Large Hadron Collider (LHC). However, the recent Higgs results have interesting implications for the SUSY parameter space. In this paper, we study the consequences of an LHC Higgs signal for a model with non-universal gaugino masses in the context of SU(5) unification. The gaugino mass ratios associated with the higher representations produce viable spectra that are largely inaccessible to the current LHC and direct dark matter detection experiments. Thus, in light of the Higgs results, the non-observation of SUSY is no surprise.

Keywords: Supersymmetry Phenomenology

ARXIV EPRINT: 1202.5288 


\section{Contents}

1 Introduction 1

2 Model and parameters 2

3 Constraints 3

4 Consequences of the Higgs mass 4

$5 \quad$ LHC SUSY searches 5

6 Direct detection $\quad 8$

$\begin{array}{llr}7 & \text { Summary and discussion } & 8\end{array}$

$\begin{array}{ll}\text { A Results for logarithmic prior } & 10\end{array}$

\section{Introduction}

Broken low-scale supersymmetry (SUSY) is one of the most relevant candidates of new physics. It may explain the hierarchy problem [1-4] and facilitates a unification of the running gauge couplings at the grand unification (GUT) scale [5-8]. In the minimal supersymmetric standard model (MSSM) with $R$-parity [9-13] imposed, it can also provide an appropriate dark matter (DM) particle. At the same time, SUSY has to reproduce known physics at lower energies. In this light, the recent signs of a possible Higgs boson at the Large Hadron Collider (LHC) $[14,15]$ can put tight constraints on supersymmetry.

The number of free parameters in the MSSM is notoriously large, leading to a loss of predictability. Therefore, the MSSM parameter space is usually restricted by a combination of theoretical and phenomenological considerations. This has lead to a study of simplified models such as the constrained MSSM (CMSSM), which has a universal scalar mass $m_{0}$ and trilinear parameter $A_{0}$ at the GUT scale as well as universal gaugino masses. However, why would the ratios of the different gaugino masses be unity at the GUT scale? A close inspection of the structure of the SUSY breaking terms in fact shows that different ratios arise quite naturally. As an example we will consider a model of non-universal gaugino masses $[16,17]$ in the context of $\mathrm{SU}(5)$ unification. Note that the $\mathrm{SU}(5)$ group may be embedded into a larger GUT group, like $\mathrm{SO}(10)$ or $\mathrm{E}(6)$ [18], in which case the studied gaugino mass ratios belong to the simplest branching rules of these embeddings.

The effect of the usual low-energy and DM constraints on non-universal gaugino mass models has been discussed in [18-36]. We will focus on the impact of the recent LHC Higgs results and the effects of the ATLAS squark and gluino searches. Finally, we will study how this affects direct DM detection. 


\begin{tabular}{|c|ccc|ccc|}
\hline rep & $M_{1}$ & $M_{2}$ & $M_{3}$ & $M_{1}^{\mathrm{EW}}$ & $M_{2}^{\mathrm{EW}}$ & $M_{3}^{\mathrm{EW}}$ \\
\hline $\mathbf{1}$ & 1 & 1 & 1 & 0.14 & 0.29 & 1 \\
$\mathbf{2 4}$ & -0.5 & -1.5 & 1 & -0.07 & -0.43 & 1 \\
$\mathbf{7 5}$ & -5 & 3 & 1 & -0.72 & 0.87 & 1 \\
$\mathbf{2 0 0}$ & 10 & 2 & 1 & 1.44 & 0.58 & 1 \\
\hline
\end{tabular}

Table 1. Ratios of the gaugino masses at the GUT scale in the normalization $M_{3}=1$, and at the EW scale in the normalization $M_{3}^{\mathrm{EW}}=1$ at the 1-loop level [24].

\section{Model and parameters}

Many supergravity-type models use unified gaugino masses at the GUT scale, but there is no compelling reason to do so. In general, the mass-generating terms for the gauginos $\lambda$ have the form $[16,37,38]$ :

$$
\mathcal{L}_{\text {gaugino mass }} \sim\left\langle F_{a b}\right\rangle \lambda^{a} \lambda^{b}+\text { c.c. } .
$$

The Lagrangian must be invariant under the gauge symmetry group, which we take to be $\mathrm{SU}(5)$. Gauginos, like the corresponding gauge bosons, reside in the adjoint representation of the gauge group, which in $\mathrm{SU}(5)$ is 24-dimensional. The gaugino product in eq. (2.1) transforms under $\mathrm{SU}(5)$ according to a representation appearing in the symmetric product of two adjoint representations:

$$
(\mathbf{2 4} \otimes \mathbf{2 4})_{S y m m}=\mathbf{1} \oplus \mathbf{2 4} \oplus \mathbf{7 5} \oplus \mathbf{2 0 0} .
$$

The mass-generating term $F_{a b}$, which contains a vacuum expectation value (vev), must be in the same representation as the gaugino product in order to make the Lagrangian invariant. Therefore, it can be in any of the representations on the right-hand side of eq. (2.2). The representation 1 corresponds to the CMSSM, where the generated gaugino masses are unified at the GUT scale. Any of the other representations, however, will yield different mass relations at the GUT scale $[16,17,19,20]$. Such different mass relations occur naturally in a GUT and have important consequences for SUSY phenomenology. Table 1 shows the ratios of resulting gaugino masses at tree-level at the GUT scale and at one-loop level at the electroweak (EW) scale. We study the case of each representation separately, although an arbitrary combination of these is also allowed (see, e.g., $[28,32,36]$ ).

In our scans of the parameter space, we sample $M_{3}$ at the GUT scale. This variable correlates with the gluino mass and fixes the other gaugino masses for each representation according to table 1 . In addition, we vary the scalar mass $m_{0}$, the trilinear parameter $A_{0}$, the ratio of the Higgs vev's $\tan \beta=\left\langle H_{u}^{0}\right\rangle /\left\langle H_{d}^{0}\right\rangle$, and the sign of the SUSY Higgs mass parameter $\mu$. The parameter space is sampled quasi-randomly $[39,40]$ using a flat prior in all variables. We have checked that the results are unchanged for a logarithmic prior and provide the relevant figures in appendix A. For each representation, we sampled 150.000 points. The scan ranges are given in table 2 .

The particle spectrum was calculated using SOFTSUSY (v.3.1.7) [41]. A top pole mass of $m_{t}=173.3 \mathrm{GeV}$ was used throughout this study. 


\begin{tabular}{|c|c|}
\hline parameter & range \\
\hline$m_{0}, M_{3}(\mathrm{GeV})$ & {$[100,3000]$} \\
$A_{0}(\mathrm{TeV})$ & {$[-7,7]$} \\
$\tan \beta$ & {$[2.5,59]$} \\
$\mu$ & \pm 1 \\
\hline
\end{tabular}

Table 2. Parameter ranges.

\section{Constraints}

After sampling the parameter space, we select viable models by requiring they satisfy a number of constraints. We will briefly list these constraints, which have all been implemented at 95\% CL using micrOmegas (v.2.4.1) [42].

Combining the present experimental value of the $B \rightarrow X_{s} \gamma$ branching ratio [43] with the theoretical uncertainties [44, 45], gives [46]:

$$
B R\left(B \rightarrow X_{s} \gamma\right)=(355 \pm 142) \times 10^{-6} .
$$

The $b \rightarrow s \gamma$ constraint is sensitive to the sign of $\mu$ [47], preferring the positive value.

We also use the $B \rightarrow \tau \nu$ branching ratio as a constraint by demanding [48, 49]:

$$
0.99<\frac{\mathrm{BR}\left(B^{+} \rightarrow \tau^{+} \nu_{\tau}\right) \mathrm{SUSY}}{\operatorname{BR}\left(B^{+} \rightarrow \tau^{+} \nu_{\tau}\right)_{\mathrm{SM}}}<3.19
$$

where the numerator denotes the branching ratio in the SUSY scenario, including the SM contribution. The constraint (3.2) tends to prefer small values of $\tan \beta$ in order not to decrease the ratio too much below the lower limit.

Furthermore, we use a conservative upper limit of $B R\left(B_{s} \rightarrow \mu^{+} \mu^{-}\right)<5.0 \times 10^{-8}$.

From the 7-year Wilkinson Microwave Anisotropy Probe (WMAP) results, the cold DM relic density in the universe [50] with a $10 \%$ theoretical uncertainty added [51], is given by

$$
0.0941<\Omega_{c} h^{2}<0.131
$$

Since DM may be of non-supersymmetric origin, we only impose the upper limit of constraint (3.3). This severely reduces the number of viable models for the $\mathbf{1}$ and the $\mathbf{2 4}$ representations, where the LSP is generally Bino-like. In contrast, in the $\mathbf{7 5}$ representation the LSP can also be Higgsino-like, while in the $\mathbf{2 0 0}$ representation, the LSP is either Winoor Higgsino-like. For such models, the annihilation cross section in the early universe is higher, naturally leading to a lower DM relic density. Whereas points with a Bino-like LSP are heavily constrained by the DM relic density, points with a Wino- or Higgsino-like LSP thus tend to survive the DM constraint.

Because of the lack of consensus on how to treat the anomalous magnetic moment of the muon [52], we have opted not to include it as a constraint. We do, however, take into account the SUSY particle mass limits obtained by LEP as implemented in micrOmegas. 


\section{Consequences of the Higgs mass}

The recent LHC results have excluded a Higgs boson with a mass beyond $127 \mathrm{GeV}$. They do, however, show an excess in the mass range 121-127 GeV. Encouraged by these results, we study the consequences of a Higgs boson with a mass between $121 \mathrm{GeV} \leq m_{H} \leq 127 \mathrm{GeV}$. As this is quite a high mass for a SUSY Higgs, one would expect that this constraint limits the parameter space. In particular, such a high mass requires sizeable corrections from the third generation squarks, thus requiring them to be heavy. Therefore, we will first study the effect of this constraint on the stop masses. Secondly, we will discuss the consequences for squarks and gluinos, since these particles play a crucial role in the SUSY searches at the LHC.

In this section, we will show how the parameter space changes due to the Higgs mass constraint. In all plots, the light grey boxes indicate the number of models in a given bin that have a neutralino LSP, with a bigger box standing for a larger number of models. In the same way, the dark grey boxes indicate how many models pass the low-energy and DM constraints listed in section 3 . The black points are models that also have the correct value of the Higgs mass.

Figure 1 shows the distribution of the two stop masses for the different representations.

By definition, the $\tilde{t}_{1}$ is lighter than the $\tilde{t}_{2}$, resulting in the lower bound in the plot. The points near the upper left edge of the plots have a large difference between the two stop masses and thus have the strongest stop mixing.

For the $\mathbf{1}$ and $\mathbf{2 4}$ representations, the constraints from section 3 conspire to exclude the largest stop masses. These representations generally have a Bino-like LSP, which tends to yield a too high DM relic density. After applying the DM constraint, the only surviving points with high stop masses have a large $\tan \beta$ value and are excluded by the $\operatorname{BR}\left(B^{+} \rightarrow\right.$ $\tau^{+} \nu_{\tau}$ ) constraint. As expected, a relatively high Higgs mass excludes a scenario where both stops are light. However, the DM constraints favour light scalars to accommodate coannihilation. Indeed, after implementing the Higgs limits, the surviving points are in the stop and stau coannihilation region, where the stops or the staus are quite heavily mixed.

The situation is quite different for the $\mathbf{7 5}$ and $\mathbf{2 0 0}$ dimensional representations. In this case, the large values of $M_{1}$ and $M_{2}$ (cf. table 1) naturally result in higher stop masses after renormalization-group running. In addition, the DM relic density is less constraining for Wino and Higgsino-like LSPs, so the constraints from section 3 still allow for large stop masses. As expected, the Higgs mass excludes both stops being light for these representations as well. This is a fairly general feature of SUSY models, which is not specific to non-universal gaugino mass models.

Summarizing the conclusions we can draw from figure 1, we see for all representations that a relatively heavy Higgs excludes the lowest stop masses, although the lightest stop can still be well below $1 \mathrm{TeV}$ due to strong stop mixing.

Figure 2 shows the masses of the lightest light-flavoured squark and the gluino. The almost diagonal upper bound in the plots comes from the influence of the gluino mass on the squark mass renormalization group running. The lower bound of the plot is a result of our scan range for $m_{0}$. 

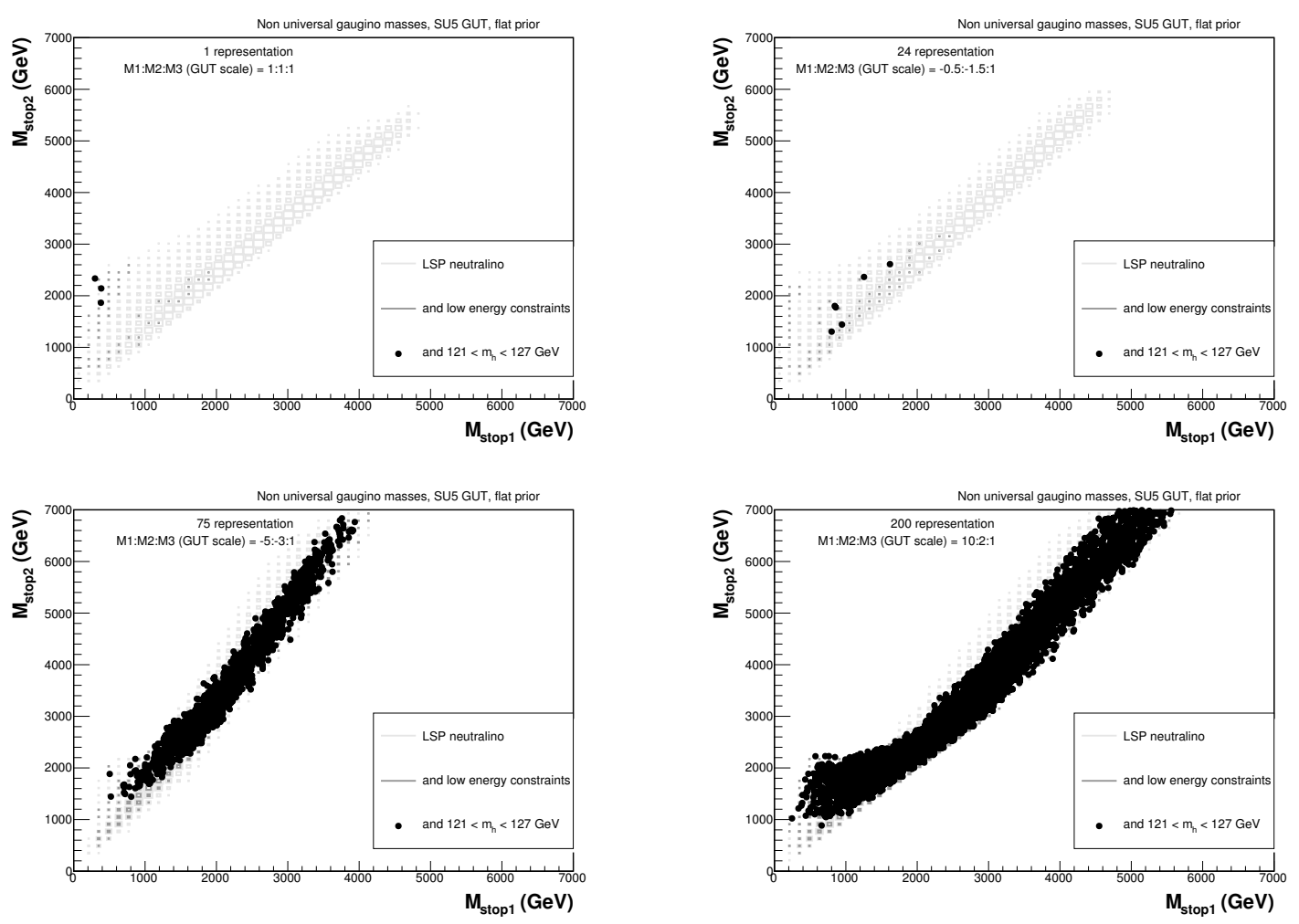

Figure 1. Stop masses in the different representations for the flat prior.

We see that after implementing the Higgs constraint, the only points left have squark masses above $1 \mathrm{TeV}$. The reason for this is that the stop mass and the squark mass are linked to each other through $m_{0}$. As we saw in figure 1, our Higgs range selects relatively heavy stops. This naturally implies that small squark masses are excluded. In the $\mathbf{1}$ and $\mathbf{2 4}$ representations, that also results in a large gluino mass. Thus for this relatively large Higgs mass, squarks and gluinos would not have been detected at the LHC yet, a conclusion that was also reached for the $\mathbf{2 4}$ representation in the context of $\mathrm{SO}(10)$ unification [53].

For the $\mathbf{7 5}$ and $\mathbf{2 0 0}$ representations, the Higgs constraint also tends to prefer higher squark masses due to the need for a higher stop mass. This kind of squark spectrum correlation is a general feature of models with universal scalar masses. However, due to the different ratios of gaugino masses and their effect on the renormalization-group running, we still have some points left with low gluino masses. Such points are very interesting in the context of direct SUSY searches. In the next section, we will investigate whether they could be detected by the LHC.

\section{LHC SUSY searches}

In this section we study the possibility that the low mass SUSY points found in the previous section are already excluded by the LHC experiments. The mass limits obtained by the LHC experiments, are either model-dependent, or quite weak. In order to assess which 

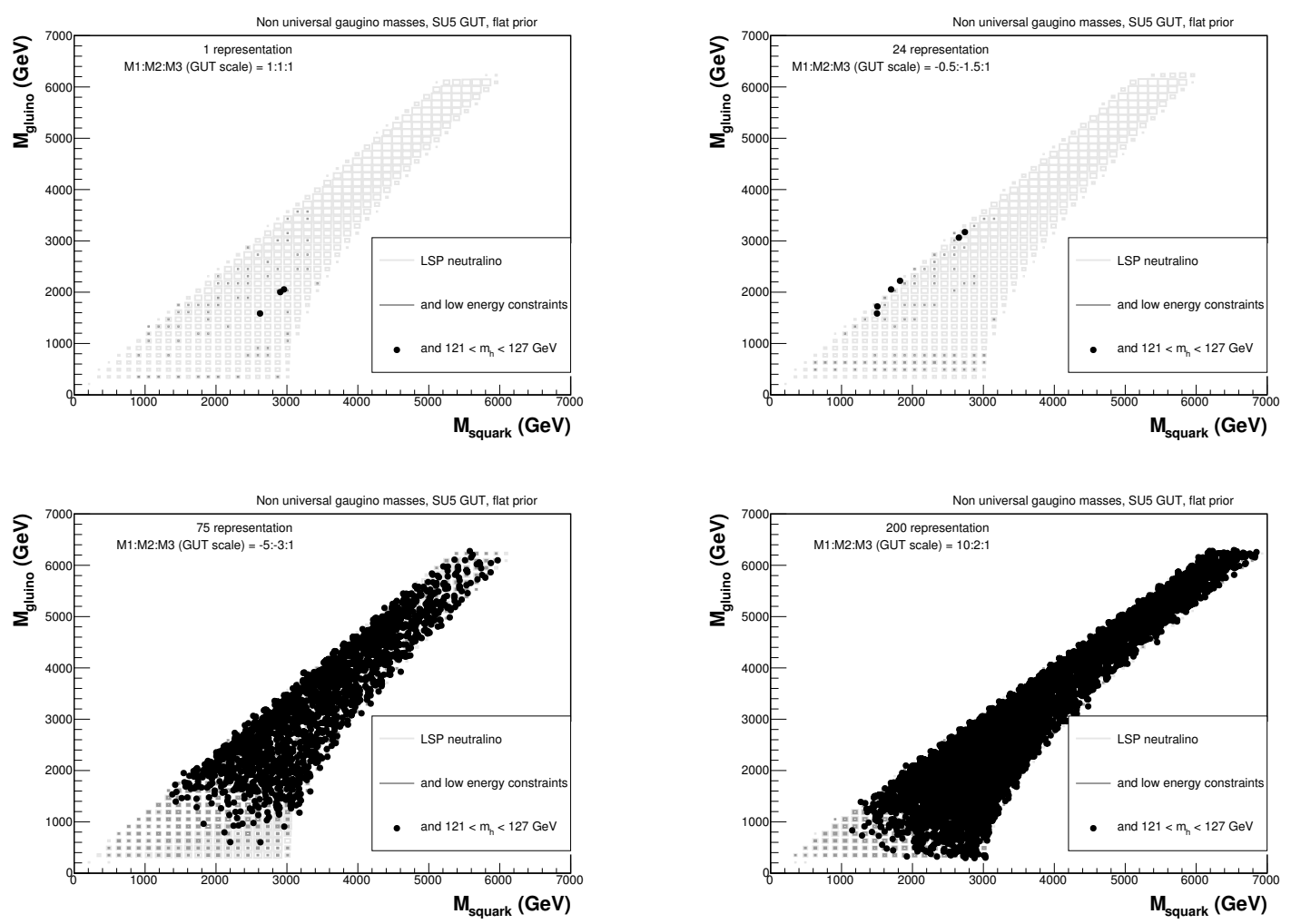

Figure 2. Squark and gluino masses in the different representations for the flat prior.

surviving points would have been detected by the LHC, we have run the models with either a first or second generation squark or a gluino mass below $1300 \mathrm{GeV}$ through an emulation of the jet + missing $E_{T}$ SUSY searches in ATLAS $[54,55]$.

Events have been generated for each SUSY model with PYTHIA 6.4 [56]. We use DELPHES 1.9 [57] as a fast detector simulation with the default ATLAS detector card, modified by setting the jet cone radius to 0.4, which is the value used in the ATLAS SUSY searches. The ATLAS analysis is implemented as in ref. [58], where it is shown that there is good agreement between the ATLAS and DELPHES setup. Since we are only making an estimate of the exclusion potential, we have not included theoretical uncertainties, even though they would lead to somewhat weaker limits. The event numbers are scaled to the integrated luminosity of the ATLAS experiment with NLO cross sections calculated using PROSPINO2.1 [59-61]. These numbers are then used to calculate the expected number of signal events for each signal region and analysis. The results are compared to the modelindependent $95 \%$ C.L. limits provided by ATLAS. Models yielding more events in one of the signal regions are called 'excluded'. Models yielding less events in all signal regions are called 'non-excluded'.

Figure 3 shows the results of this study in the squark-gluino mass plane on the lefthand side. On the right-hand side, the same points are shown as a function of $M_{S U S Y}$, which is defined as the lightest of the gluino and the light-flavoured squarks, and the mass splitting between this particle and the neutralino. The $\mathbf{7 5}$ representation is shown in the 

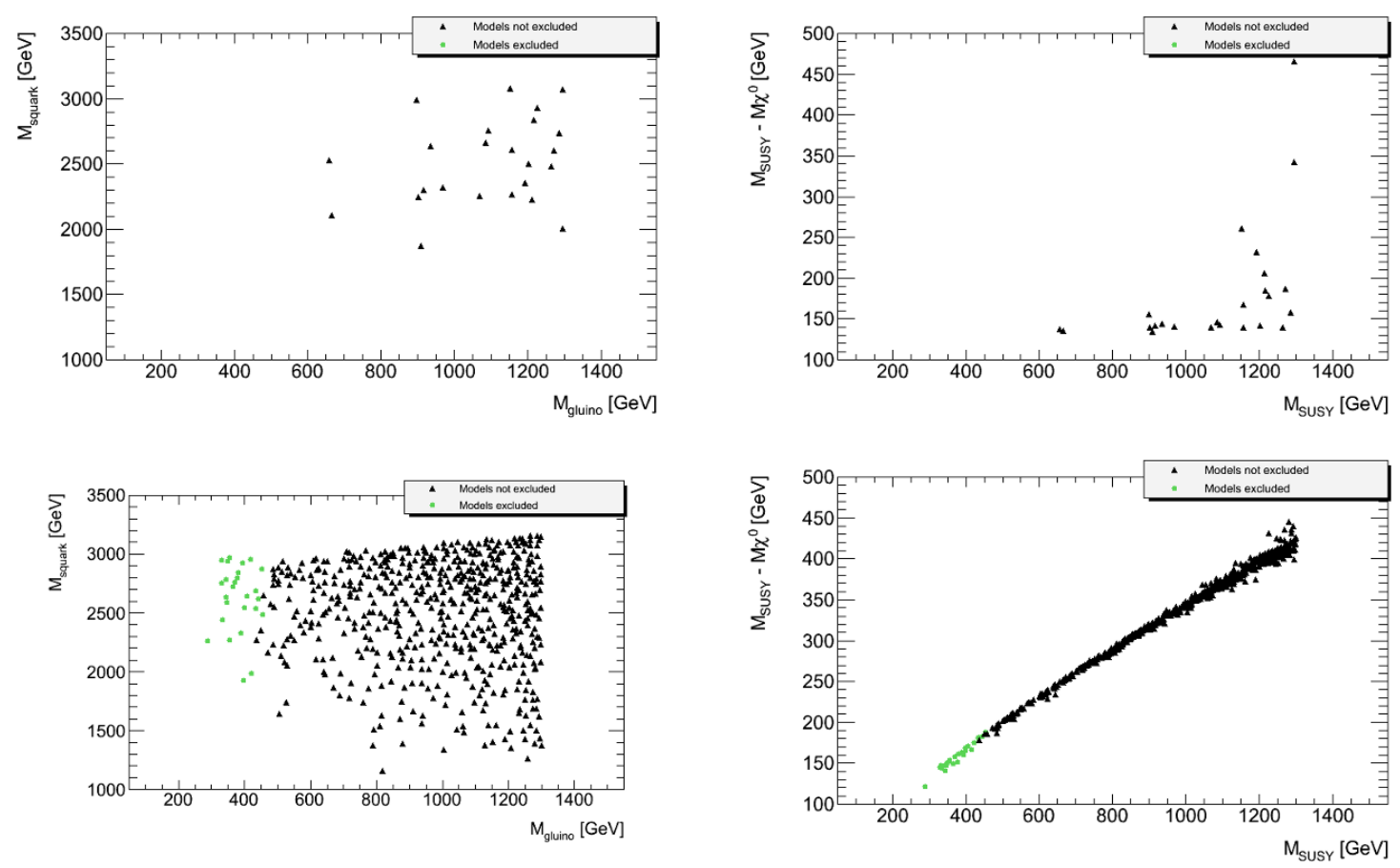

Figure 3. The effect of the LHC searches on the surviving low-mass points. The representation $\mathbf{7 5}$ is shown in the top row, while the $\mathbf{2 0 0}$ representation is shown in the bottom row.

top row and the $\mathbf{2 0 0}$ representation in the lower row. The black points are not excluded by the LHC, while the green points are.

The shape of gluino-LSP mass splitting is quite different for the two representations. This is caused by the composition of the LSP. In the $\mathbf{7 5}$ representation, the points with $M_{\mathrm{SUSY}}-m_{\widetilde{\chi}_{1}^{0}} \leq 160 \mathrm{GeV}$ correspond to a Bino-type neutralino, while the other points correspond to a Higgsino-type neutralino. In the $\mathbf{2 0 0}$ representation, all the low-mass points correspond to Wino-type neutralinos.

We see that no models are excluded for the $\mathbf{7 5}$ representation. For the $\mathbf{2 0 0}$ representation, models with gluino masses below $400 \mathrm{GeV}$ are excluded by the ATLAS searches. Models with gluino masses above $400 \mathrm{GeV}$ are not excluded. The reason that such low gluino masses are not excluded in these representations is that the mass splittings in these models are small. As can be seen in figure 3, the neutralinos tend to be quite heavy compared to the gluino mass. One would need a dedicated search for gluinos with a small or moderate mass difference with the neutralino to exclude these models at the LHC. For part of the model points, the gluino can decay to a stop and a top. In some cases, the subsequent stop decays can produce a total of four tops, leading to spectacular events in the detector.

Note that the gluino can still escape detection if the mass difference is as large as several hundred $\mathrm{GeV}$. Thus, this problem is not limited to extremely fine-tuned scenarios, but would occur in many models with non-unified masses. A dedicated search for relatively small mass splittings could therefore be useful for a much wider range of SUSY models. 


\section{Direct detection}

Several direct detection experiments aim at measuring the recoil energy of the nuclei from an elastic dark matter - nucleus collision. At present, the most stringent limits to the spin independent elastic cross section for high weakly-interacting massive particle (WIMP) masses come from the XENON100 experiment [62]. To compare the calculated proton/neutron cross sections to the experimental limits, we use a normalized cross section for a point-like nucleus [63]:

$$
\sigma_{\mathrm{SI}}=\frac{\left(Z \sqrt{\sigma_{\mathrm{SI}}^{p}}+(A-Z) \sqrt{\sigma_{\mathrm{SI}}^{n}}\right)^{2}}{A^{2}},
$$

$Z$ and $A$ being the atomic and mass number of the target element. Because there are large uncertainties in the local density of dark matter and in the nuclear matrix elements that enter the computation of $\sigma_{\mathrm{SI}}[64,65]$, the direct detection limits are only indicative.

Figure 4 shows the neutralino mass and the spin independent cross section for the different representations as well as the XENON100 limit. Once again, the light-grey boxes indicate how many models have a neutralino LSP, the dark grey boxes show the number of models that survive the constraints from section 3 and the black points correspond to models that have the correct Higgs mass as well.

We see that most of the models that survive all the constraints from section 3 and have the correct Higgs mass cannot be detected by the current direct DM experiments. Only for the $\mathbf{2 0 0}$ representation, some of the otherwise allowed points could be excluded by the XENON100 limit. For the other representations, the surviving points are well below the direct detection limits, and the corresponding models are in accordance with all current experimental constraints.

Although the neutralino-nucleon cross section heavily depends on the specific SUSY parameters, the next generation of experiments would be able to detect a large part of the surviving parameter space from the two higher representations. Also, since high neutralino masses are a viable option for Wino and Higgsino LSPs, it would be interesting to extend the XENON100 limit to masses beyond $1 \mathrm{TeV}$.

\section{$7 \quad$ Summary and discussion}

We have studied the consequences of the recent signs of a Higgs with a mass of 121$127 \mathrm{GeV}$ for SU(5) GUT models with non-universal gaugino masses. We find that results for the different representations can be quite different. We can, however, draw some general conclusions. Models where both stops are light are excluded by the Higgs constraint. As a result, squarks with masses below $1 \mathrm{TeV}$ are excluded as well due to their intimiate connection to stops in models with unified scalar masses. Light gluinos, however, are still an option. These could arise from several models without gaugino mass unification.

We have studied the surviving models with low gluino masses in more detail to see if they would have been observed by the ATLAS direct SUSY searches. We find that indeed some models are excluded. However, for the $\mathbf{2 0 0}$ representation, even gluinos with masses 

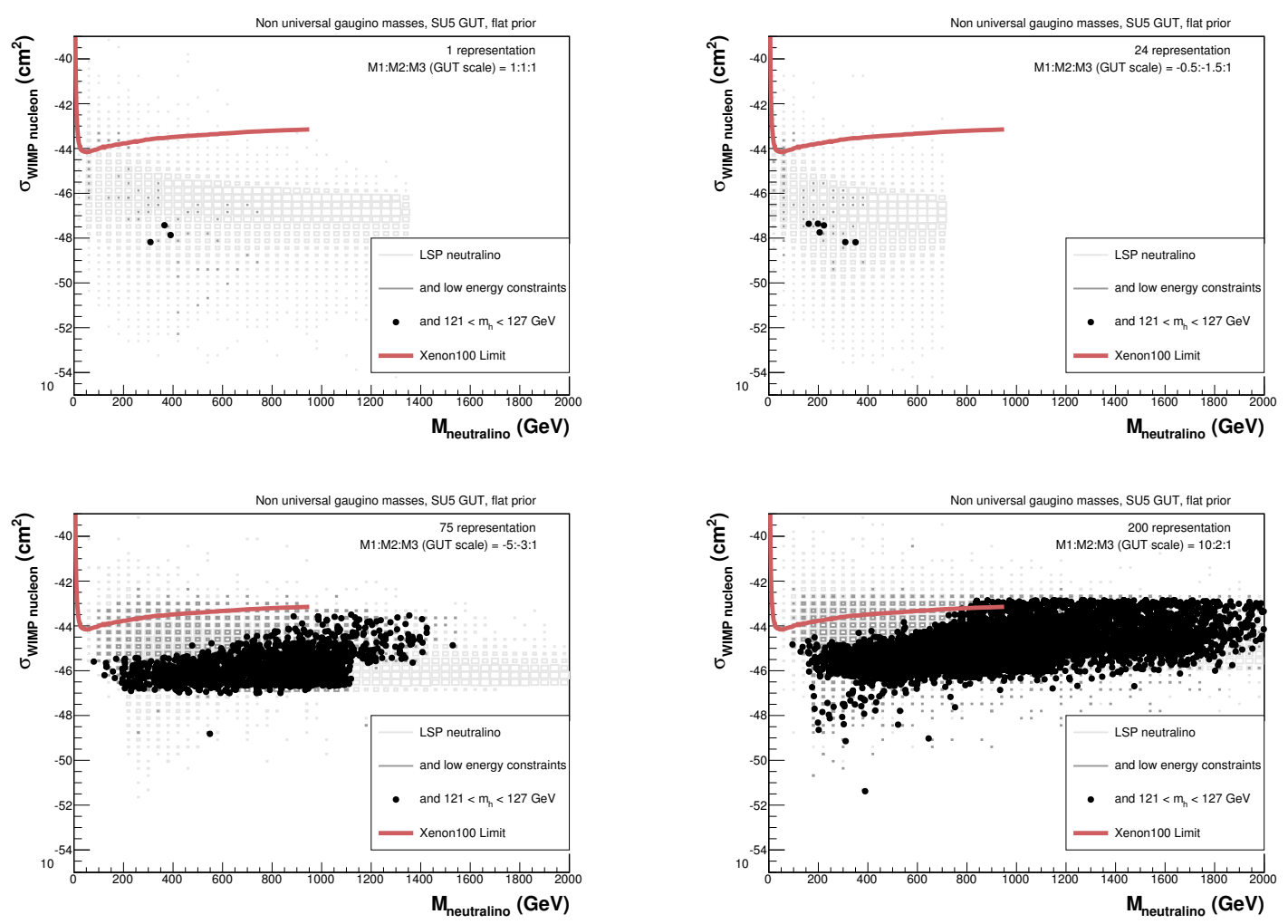

Figure 4. Spin-independent WIMP-nucleon cross section for the different representations for the flat prior.

below $500 \mathrm{GeV}$ can escape detection due to the small gluino-LSP mass splitting. To detect such models at the LHC, one would most likely need a dedicated search for models with small or moderate mass splittings between the SUSY particles. Such mass splittings could arise in many SUSY scenarios and are not specific for non-universal gaugino mass models.

Finally, we have studied the effect of the Higgs constraint on direct DM detection. We find that if the Higgs is heavy, the current exclusion limits are not sensitive to the largest part of the parameter space. Future experiments would be able to detect many of the interesting points. Also, it would be interesting to extend the range of these searches to higher neutralino masses.

We can conclude that the signs of a heavy Higgs point towards a heavy SUSY mass scale, particularly in the scalar sector. More importantly, most of the points that survive the low-energy, DM and Higgs constraints would not have been detected by the LHC or direct DM searches. Thus, in light of the Higgs results, the non-observation of SUSY so far is no surprise.

\section{Acknowledgments}

We thank Wim Beenakker for useful discussions. The work of JL and IN was supported by the Foundation for Fundamental Research of Matter (FOM), program 104 "Theoretical Particle Physics in the Era of the LHC". 

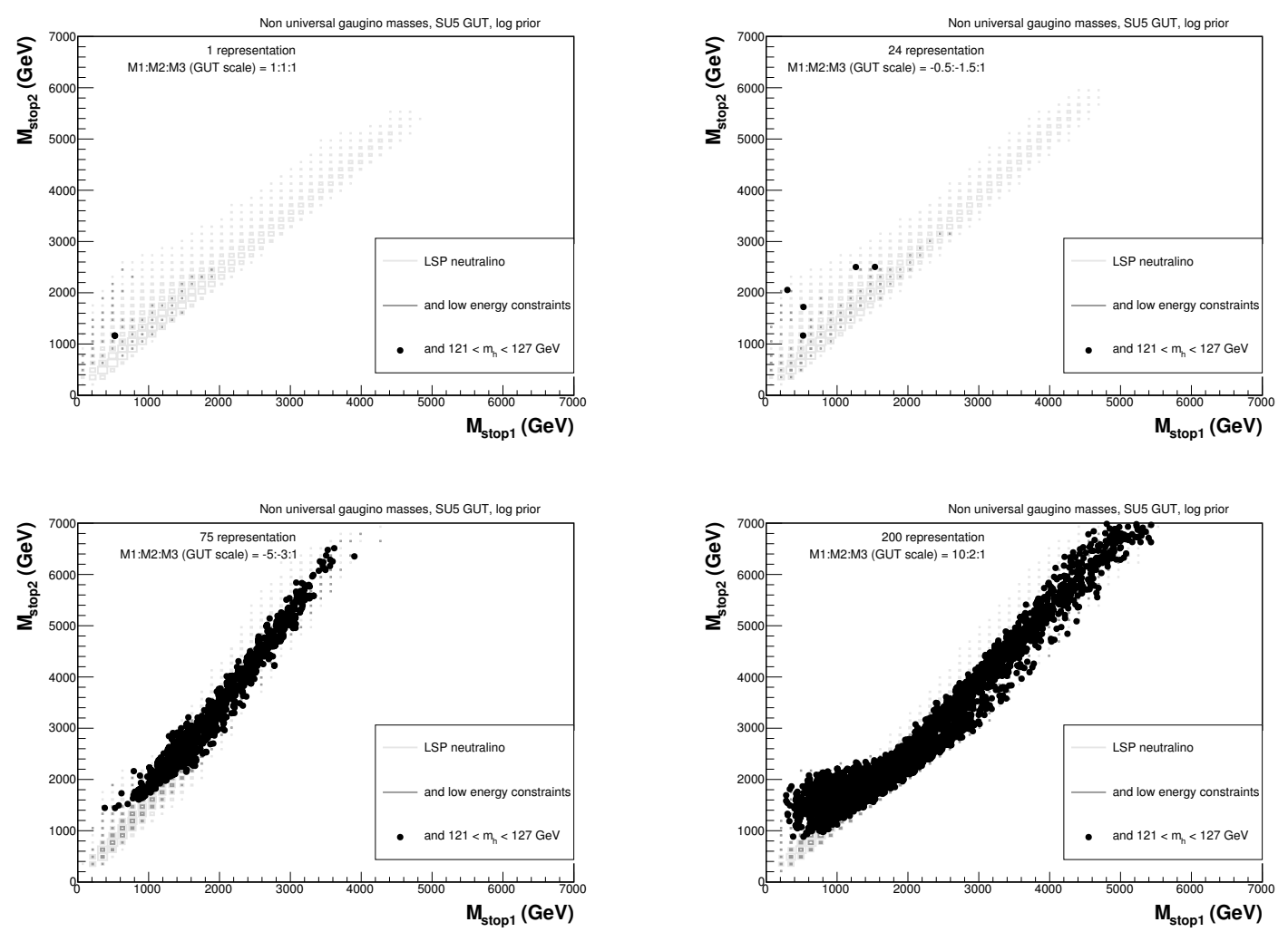

Figure 5. Stop masses in the different representations for the log prior.

\section{A Results for logarithmic prior}

In this appendix, we show the plots for the logarithmic prior. The ranges of this prior are the same as those for the flat prior, but the variables $m_{0}$ and $M_{3}$ are distributed logarithmically.

Figure 5 shows the results for the stop masses. We see some minor changes because the logarithmic prior emphasizes the low mass range, but the main conclusions remain unchanged: not both stops can be light, but the lightest stop can be considerably lighter than $1 \mathrm{TeV}$ due to mixing.

Figure 6 shows the squark-gluino mass plane. We see once again that light lightflavoured squarks are excluded, but that light gluinos are still a viable option.

Open Access. This article is distributed under the terms of the Creative Commons Attribution Noncommercial License which permits any noncommercial use, distribution, and reproduction in any medium, provided the original author(s) and source are credited. 

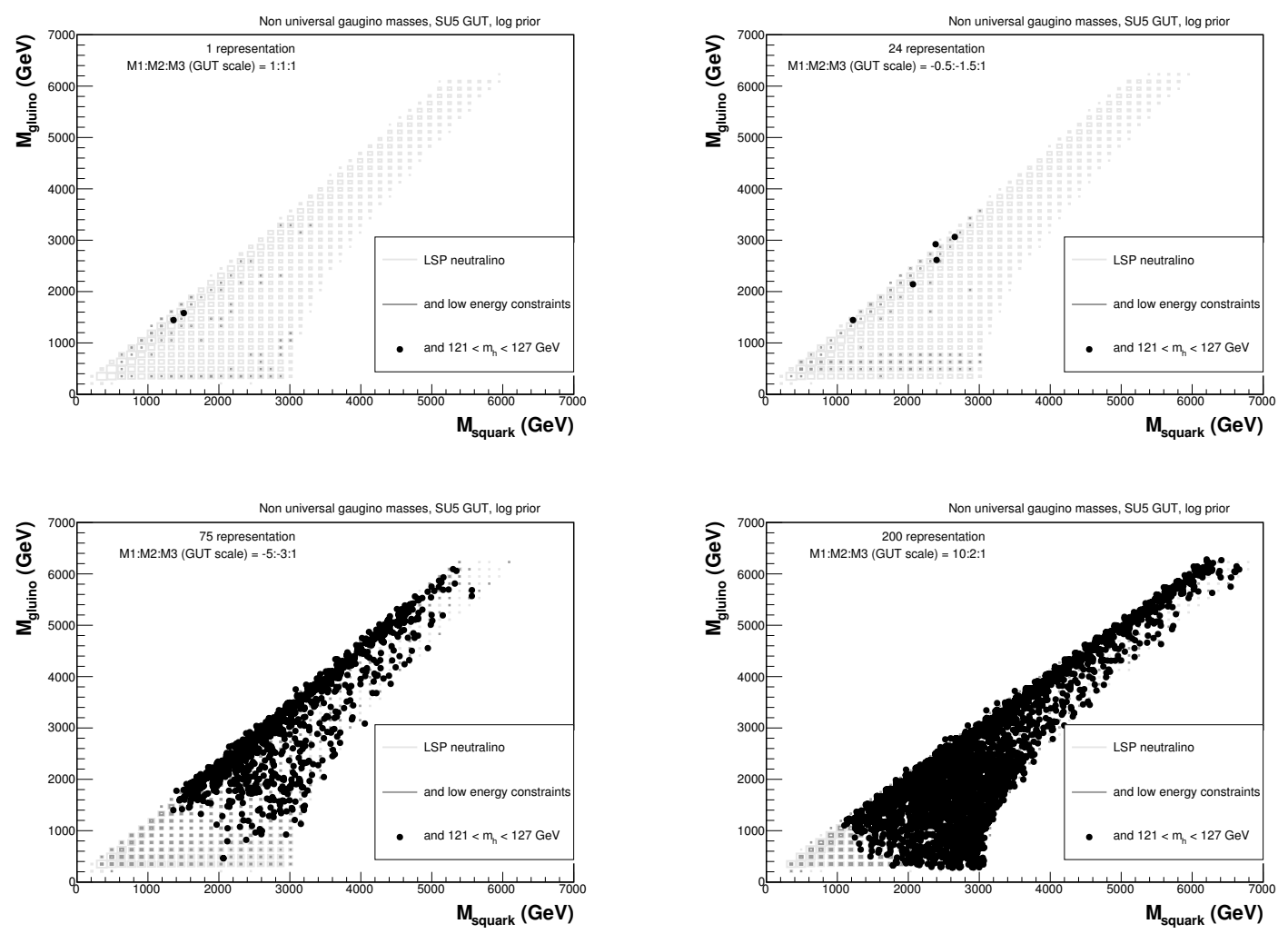

Figure 6. Squark and gluino masses in the different representations for the log prior.

\section{References}

[1] E. Witten, Dynamical breaking of supersymmetry, Nucl. Phys. B 188 (1981) 513 [InSPIRE].

[2] S. Dimopoulos and H. Georgi, Softly broken supersymmetry and SU(5), Nucl. Phys. B 193 (1981) 150 [INSPIRE].

[3] N. Sakai, Naturalness in supersymmetric guts, Z. Phys. C 11 (1981) 153 [INSPIRE].

[4] R.K. Kaul and P. Majumdar, Cancellation of quadratically divergent mass corrections in globally supersymmetric spontaneously broken gauge theories, Nucl. Phys. B 199 (1982) 36 [INSPIRE].

[5] J.R. Ellis, S. Kelley and D.V. Nanopoulos, Probing the desert using gauge coupling unification, Phys. Lett. B 260 (1991) 131 [INSPIRE].

[6] C. Giunti, C. Kim and U. Lee, Running coupling constants and grand unification models, Mod. Phys. Lett. A 6 (1991) 1745 [inSPIRE].

[7] U. Amaldi, W. de Boer and H. Furstenau, Comparison of grand unified theories with electroweak and strong coupling constants measured at LEP, Phys. Lett. B 260 (1991) 447 [INSPIRE].

[8] P. Langacker and M.-x. Luo, Implications of precision electroweak experiments for $M_{t}, \rho_{0}$, $\sin ^{2} \theta_{W}$ and grand unification, Phys. Rev. D 44 (1991) 817 [INSPIRE]. 
[9] A. Salam and J. Strathdee, Supersymmetry and fermion number conservation, Nucl. Phys. B 87 (1975) 85 [INSPIRE].

[10] P. Fayet, Supergauge invariant extension of the Higgs mechanism and a model for the electron and its neutrino, Nucl. Phys. B 90 (1975) 104 [InSPIRE].

[11] G.R. Farrar and P. Fayet, Phenomenology of the production, decay and detection of new hadronic states associated with supersymmetry, Phys. Lett. B 76 (1978) 575 [INSPIRE].

[12] S. Dimopoulos, S. Raby and F. Wilczek, Proton decay in supersymmetric models, Phys. Lett. B 112 (1982) 133 [INSPIRE].

[13] G.R. Farrar and S. Weinberg, Supersymmetry at ordinary energies. 2. $R$ invariance, Goldstone bosons, and gauge fermion masses, Phys. Rev. D 27 (1983) 2732 [InSPIRE].

[14] ATLAS collaboration, G. Aad et al., Combined search for the standard model Higgs boson using up to $4.9 \mathrm{fb}^{-1}$ of pp collision data at $\sqrt{\mathrm{s}}=7 \mathrm{TeV}$ with the ATLAS detector at the LHC, Phys. Lett. B 710 (2012) 49 [arXiv:1202.1408] [inSPIRE].

[15] CMS collaboration, S. Chatrchyan et al., Combined results of searches for the standard model Higgs boson in pp collisions at $\sqrt{s}=7$ TeV, Phys. Lett. B 710 (2012) 26 [arXiv:1202.1488] [INSPIRE].

[16] J.R. Ellis, K. Enqvist, D.V. Nanopoulos and K. Tamvakis, Gaugino masses and grand unification, Phys. Lett. B 155 (1985) 381 [INSPIRE].

[17] M. Drees, Phenomenological consequences of $N=1$ supergravity theories with nonminimal kinetic energy terms for vector superfields, Phys. Lett. B 158 (1985) 409 [INSPIRE].

[18] S.P. Martin, Non-universal gaugino masses from non-singlet F-terms in non-minimal unified models, Phys. Rev. D 79 (2009) 095019 [arXiv:0903.3568] [INSPIRE].

[19] G. Anderson et al., Motivations for and implications of nonuniversal GUT scale boundary conditions for soft SUSY breaking parameters, eConf C 960625 (1996) SUP107 [hep-ph/9609457] [INSPIRE].

[20] G. Anderson, H. Baer, C.-h. Chen and X. Tata, The reach of Fermilab Tevatron upgrades for $\mathrm{SU}(5)$ supergravity models with nonuniversal gaugino masses, Phys. Rev. D 61 (2000) 095005 [hep-ph/9903370] [INSPIRE].

[21] K. Huitu, Y. Kawamura, T. Kobayashi and K. Puolamaki, Phenomenological constraints on SUSY SU(5) GUTs with nonuniversal gaugino masses, Phys. Rev. D 61 (2000) 035001 [hep-ph/9903528] [INSPIRE].

[22] A. Corsetti and P. Nath, Gaugino mass nonuniversality and dark matter in SUGRA, strings and D-brane models, Phys. Rev. D 64 (2001) 125010 [hep-ph/0003186] [INSPIRE].

[23] A. Birkedal-Hansen and B.D. Nelson, Relic neutralino densities and detection rates with nonuniversal gaugino masses, Phys. Rev. D 67 (2003) 095006 [hep-ph/0211071] [INSPIRE].

[24] K. Huitu, J. Laamanen, P.N. Pandita and S. Roy, Phenomenology of non-universal gaugino masses in supersymmetric grand unified theories, Phys. Rev. D 72 (2005) 055013 [hep-ph/0502100] [INSPIRE].

[25] G. Bélanger, F. Boudjema, A. Cottrant, A. Pukhov and A. Semenov, WMAP constraints on SUGRA models with non-universal gaugino masses and prospects for direct detection, Nucl. Phys. B 706 (2005) 411 [hep-ph/0407218] [INSPIRE]. 
[26] B. Ananthanarayan and P. Pandita, Sparticle mass spectrum in grand unified theories, Int. J. Mod. Phys. A 22 (2007) 3229 [arXiv:0706.2560] [INSPIRE].

[27] S. Bhattacharya, A. Datta and B. Mukhopadhyaya, Non-universal gaugino masses: a signal-based analysis for the Large Hadron Collider, JHEP 10 (2007) 080 [arXiv:0708.2427] [INSPIRE].

[28] K. Huitu, R. Kinnunen, J. Laamanen, S. Lehti, S. Roy, et al., Search for Higgs bosons in SUSY cascades in CMS and dark matter with non-universal gaugino masses, Eur. Phys. J. C 58 (2008) 591 [arXiv:0808.3094] [INSPIRE].

[29] M. Drees and J.M. Kim, Neutralino dark matter in an $\mathrm{SO}(10)$ model with two-step intermediate scale symmetry breaking, JHEP 12 (2008) 095 [arXiv:0810.1875] [INSPIRE].

[30] P. Bandyopadhyay, Probing non-universal gaugino masses via Higgs boson production under SUSY cascades at the LHC: a detailed study, JHEP 07 (2009) 102 [arXiv:0811.2537] [INSPIRE].

[31] K. Huitu and J. Laamanen, Relic density in non-universal gaugino mass models with $\mathrm{SO}(10)$ GUT symmetry, Phys. Rev. D 79 (2009) 085009 [arXiv:0901.0668] [InSPIRE].

[32] U. Chattopadhyay, D. Das and D. Roy, Mixed neutralino dark matter in nonuniversal gaugino mass models, Phys. Rev. D 79 (2009) 095013 [arXiv: 0902.4568] [inSPIRE].

[33] S. Bhattacharya and J. Chakrabortty, Gaugino mass non-universality in an $\mathrm{SO}(10)$ supersymmetric grand unified theory: low-energy spectra and collider signals, Phys. Rev. D 81 (2010) 015007 [arXiv:0903.4196] [INSPIRE].

[34] N. Okada, S. Raza and Q. Shafi, Particle spectroscopy of supersymmetric $\mathrm{SO}(10)$ with non-universal gaugino masses, Phys. Rev. D 84 (2011) 095018 [arXiv:1107.0941] [INSPIRE].

[35] X.-J. Bi, Q.-S. Yan and P.-F. Yin, Probing light stop pairs at the LHC, Phys. Rev. D 85 (2012) 035005 [arXiv: 1111.2250] [INSPIRE].

[36] J.E. Younkin and S.P. Martin, Non-universal gaugino masses, the supersymmetric little hierarchy problem and dark matter, Phys. Rev. D 85 (2012) 055028 [arXiv:1201.2989] [INSPIRE].

[37] E. Cremmer, S. Ferrara, L. Girardello and A. Van Proeyen, Coupling supersymmetric Yang-Mills theories to supergravity, Phys. Lett. B 116 (1982) 231 [InSPIRE].

[38] E. Cremmer, S. Ferrara, L. Girardello and A. Van Proeyen, Yang-Mills theories with local supersymmetry: lagrangian, transformation laws and super-Higgs effect, Nucl. Phys. B 212 (1983) 413 [INSPIRE].

[39] H. Niederreiter, Low-discrepancy and low-dispersion sequences, J. Numb. Theor. 30 (1988) 51.

[40] http://people.sc.fsu.edu/ jburkardt.

[41] B. Allanach, SOFTSUSY: a C++ program for calculating supersymmetric spectra, Comput. Phys. Commun. 143 (2002) 305 [hep-ph/0104145] [InSPIRE].

[42] G. Bélanger, F. Boudjema, A. Pukhov and A. Semenov, MicrOMEGAs 2.0: a program to calculate the relic density of dark matter in a generic model, Comput. Phys. Commun. 176 (2007) 367 [hep-ph/0607059] [INSPIRE].

[43] Heavy Flavor Averaging Group collaboration, D. Asner et al., Averages of b-hadron, c-hadron and $\tau$-lepton properties, arXiv:1010.1589 [INSPIRE]. 
[44] M. Misiak et al., Estimate of $B\left(\bar{B} \rightarrow X_{s} \gamma\right)$ at $O\left(\alpha_{s}^{2}\right)$, Phys. Rev. Lett. 98 (2007) 022002 [hep-ph/0609232] [INSPIRE].

[45] J.R. Ellis, S. Heinemeyer, K. Olive, A. Weber and G. Weiglein, The supersymmetric parameter space in light of $B^{-}$physics observables and electroweak precision data, JHEP 08 (2007) 083 [arXiv:0706.0652] [INSPIRE].

[46] K. Huitu, L. Leinonen and J. Laamanen, Stop as a next-to-lightest supersymmetric particle in constrained MSSM, Phys. Rev. D 84 (2011) 075021 [arXiv:1107.2128] [INSPIRE].

[47] P. Nath and R.L. Arnowitt, $b \rightarrow s \gamma$ decay in supergravity grand unification and dark matter, Phys. Lett. B 336 (1994) 395 [hep-ph/9406389] [INSPIRE].

[48] G. Isidori and P. Paradisi, Hints of large $\tan \beta$ in flavour physics, Phys. Lett. B 639 (2006) 499 [hep-ph/0605012] [INSPIRE].

[49] B. Bhattacherjee, A. Dighe, D. Ghosh and S. Raychaudhuri, Do new data on $\left[B^{+} \rightarrow \tau^{+} \nu_{\tau}\right]$ decays point to an early discovery of supersymmetry at the LHC?, Phys. Rev. D 83 (2011) 094026 [arXiv: 1012.1052] [INSPIRE].

[50] WMAP collaboration, E. Komatsu et al., Seven-year Wilkinson Microwave Anisotropy Probe (WMAP) observations: cosmological interpretation, Astrophys. J. Suppl. 192 (2011) 18 [arXiv: 1001.4538 ] [INSPIRE].

[51] N. Baro, F. Boudjema and A. Semenov, Full one-loop corrections to the relic density in the MSSM: a few examples, Phys. Lett. B 660 (2008) 550 [arXiv:0710.1821] [INSPIRE].

[52] S. Bodenstein, C. Dominguez and K. Schilcher, Hadronic contribution to the muon $g-2:$ a theoretical determination, Phys. Rev. D 85 (2012) 014029 [arXiv:1106.0427] [INSPIRE].

[53] M. Badziak and K. Sakurai, LHC constraints on Yukawa unification in $\mathrm{SO}(10)$, JHEP 02 (2012) 125 [arXiv:1112.4796] [INSPIRE].

[54] ATLAS collaboration, G. Aad et al., Search for squarks and gluinos using final states with jets and missing transverse momentum with the ATLAS detector in $\sqrt{s}=7 \mathrm{TeV}$ proton-proton collisions, Phys. Lett. B 701 (2011) 186 [arXiv:1102.5290] [INSPIRE].

[55] ATLAS collaboration, G. Aad et al., Search for squarks and gluinos using final states with jets and missing transverse momentum with the ATLAS detector in $\sqrt{s}=7 \mathrm{TeV}$ proton-proton collisions, Phys. Lett. B $\mathbf{7 1 0}$ (2012) 67 [arXiv:1109.6572] [INSPIRE].

[56] T. Sjöstrand, S. Mrenna and P.Z. Skands, PYTHIA 6.4 physics and manual, JHEP 05 (2006) 026 [hep-ph/0603175] [inSPIRE].

[57] S. Ovyn, X. Rouby and V. Lemaitre, DELPHES, a framework for fast simulation of a generic collider experiment, arXiv:0903.2225 [INSPIRE].

[58] A. Strubig, S. Caron and M. Rammensee, Constraints on the pMSSM from searches for squarks and gluinos by ATLAS, arXiv:1202.6244 [INSPIRE].

[59] W. Beenakker, R. Hopker, M. Spira and P. Zerwas, Squark and gluino production at hadron colliders, Nucl. Phys. B 492 (1997) 51 [hep-ph/9610490] [InSPIRE].

[60] W. Beenakker, M. Krämer, T. Plehn, M. Spira and P. Zerwas, Stop production at hadron colliders, Nucl. Phys. B 515 (1998) 3 [hep-ph/9710451] [INSPIRE].

[61] http://www.thphys.uni-heidelberg.de/ ${ }^{\sim}$ plehn/prospino/ or http://people.web.psi.ch/spira/prospino/. 
[62] XENON100 collaboration, E. Aprile et al., Dark matter results from 100 live days of XENON100 data, Phys. Rev. Lett. 107 (2011) 131302 [arXiv:1104.2549] [INSPIRE].

[63] G. Bélanger, M. Kakizaki, E. Park, S. Kraml and A. Pukhov, Light mixed sneutrinos as thermal dark matter, JCAP 11 (2010) 017 [arXiv: 1008.0580] [INSPIRE].

[64] J.R. Ellis, K.A. Olive, Y. Santoso and V.C. Spanos, Update on the direct detection of supersymmetric dark matter, Phys. Rev. D 71 (2005) 095007 [hep-ph/0502001] [INSPIRE].

[65] G. Bélanger, F. Boudjema, A. Pukhov and A. Semenov, Dark matter direct detection rate in a generic model with MicrOMEGAs 2.2, Comput. Phys. Commun. 180 (2009) 747 [arXiv:0803.2360] [INSPIRE]. 